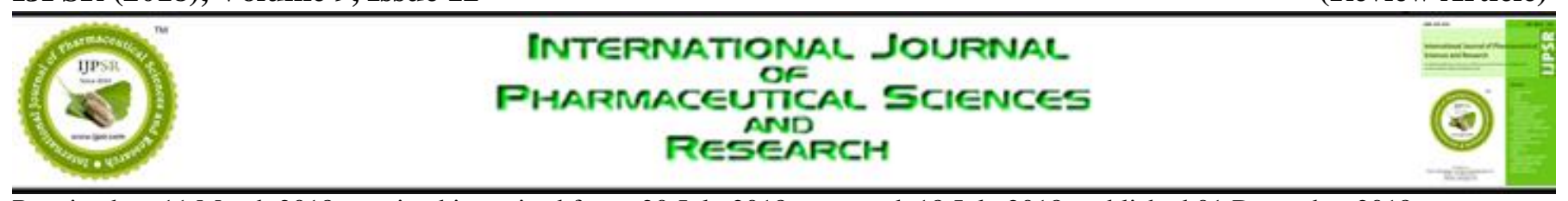

Received on 11 March 2018; received in revised form, 20 July 2018; accepted, 18 July 2018; published 01 December 2018

\title{
ASPERGILLOSIS: AN OVERVIEW
}

Deepraj Paul $^{* 1}$ and Karthika Paul ${ }^{2}$

Department of Pharmacology ${ }^{1}$, Department of Pharmaceutical Chemistry ${ }^{2}$, Vivekananda College of Pharmacy, Bengaluru - 560055, Karnataka, India.

Keywords:

Aspergillus fumigatus, Haematopoietic stem-cell transplantation, Azoles, Amphotericin $\mathrm{B}, \mathrm{PCR}, \mathrm{CT}$ scan

\section{Correspondence to Author:}

Deepraj Paul

Assistant Professor,

Department of Pharmacology, Vivekananda College of Pharmacy,

Dr. Rajkumar Road, Rajajinagar $2^{\text {nd }}$

Stage, Bengaluru - 560055,

Karnataka, India.

E-mail: deeprajcology81@gmail.com

\begin{abstract}
Aspergillus produces both airborne and waterborne infections with deadly consequences depending on the species involved and the immune status of patients. Patients may remain asymptomatic for up to 18 months. The severity of aspergillosis is mainly seen in HSCT recipients with mortality up to $90 \%$. Though aspergillosis is primarily opportunistic and lung is the primary site of infection yet deviation observed where the primary site was organs other than lungs. The major reasons behind invasion are prolonged ( $>3$ weeks) high dose corticosteroid therapy, broad-spectrum antibiotics, CMV infection, iron overload, decreased neutrophil count $(<500$ cells $/ \mathrm{mm}^{3}$ ), etc. Monoclonal technology in the form of EB-A2 and JF5 antibody can identify fungal antigen much before the appearance of clinical symptoms. Selection of a test and its success to determine fungal invasion depend on associated morbidity as galactomannan assay is more suitable in HSCT recipients rather than solid organ transplant recipients whereas PCR is specific for cancer patients with thrombocytopenia to differentiate between aspergillosis and histoplasmosis. Thorough knowledge about the neutrophil profile is required to interpret the results of CT scan and biopsy. The success of treatment depends on early and correct diagnosis as Aspergillus mimics precancerous conditions and there is considerable overlapping of symptoms between different species of Aspergillus and therapy must be species specific. Mutations in Cyp51A, L98H, M220I, F219C, and G54W are responsible for azole resistance, but the mystery of resistance did not resolve completely as certain fungal isolates show no such mutations but are resistant to antifungals; indicating the need of further research.
\end{abstract}

INTRODUCTION: Fungal invasion arising in the form of aspergillosis may cripple the life of an individual. Aspergillus primarily targets lungs and sinuses but can disseminate to other organs also ${ }^{1}$. The portals of entry of Aspergillus are a respiratory tract, damaged skin, operative wounds, cornea and ear $^{2}$.

\begin{tabular}{|l|c|}
\hline QUICK RESPONSE CODE & DOI: \\
\hline DOI link: http://dx.doi.org/10.13040/IJPSR.0975-8232.9(12).5032-49 \\
\hline
\end{tabular}

Aspergillosis specifically invasive pulmonary aspergillosis (IPA) was first reported in $1953^{3}$, a major cause of morbidity in hematopoietic stem cell recipients and in neutropenic patients 4, 5, 6 , occurs due to the mold Aspergillus which exists approximately as 250 individual species with an expectation of further increase in the count as the scientists discover new species and refine species concepts.

Only some, more than 20 out of the 250 species are toxic to human being and animals causing severe invasive infections whereas other Aspergillis cause allergies or mycoses; the majority of human illness 
is caused by Aspergillus fumigatus, Aspergillus niger and Aspergillus terreus and less frequently by Aspergillus flavus, Aspergillus clavatus and Aspergillus ustus. But there are reports of Aspergillus ustus as the emerging nosocomial pathogen particularly among transplant patients 7,8 , 9 . Aspergillosis is probably the most opportunistic fungal infection as it can wait as long as up to 18 months for a favorable condition to strike back, as can be seen with a lung transplant recipient developing endophthalmitis caused by Aspergillus fumigatus, which subsequently invaded the lungs, skin and eventually leading to the death of the patient 10

Though many pathogenic species of Aspergillus are present still Aspergillus fumigatus is found to have much higher invasion rate as high as $90 \%$ of all cases of invasive aspergillosis; maybe because of its unique ability to continue growing at much higher temperature as high as $50{ }^{\circ} \mathrm{C}$ and at very low oxygen tension $\left(0.1 \% \mathrm{O}_{2}\right)$; producing very small spore size of 3-5 $\mu^{2}$. Soubani AO et al., reported their presence in soil, rotten vegetation and hospital 11, which is the cause of severe pneumonia, invasive aspergillosis and other fungal dissemination following inhalation of fungal conidia which subsequently give rise to fungal hyphae, spreading throughout the lung parenchyma and vasculature ${ }^{12}$. Infection may also be waterborne ${ }^{13}$. In our study, we are trying to focus on different forms of Aspergillus invasion, symptoms, diagnosis and treatment.

\section{Immunological Status, its Relation to Fungal Manifestations, Other Disseminations and} Invasions: Depending upon the immunological status and/or presence of certain predisposing disease or conditions among patient population pulmonary mycosis may have several manifestations like IPA, chronic necrotizing aspergillosis, aspergilloma and allergic bronchopulmonary aspergillosis etc; manifestation in the form of IPA is found in severely immune compromised patients, critically ill patients and chronic obstructive pulmonary disease (COPD) patients; whereas locally invasive chronic necrotizing aspergillosis (CNA) reported in $1981^{14}$, is seen mainly in patients with mild immunodeficiency (due to diabetes mellitus, alcoholism, chronic liver disease, low-dose corticosteroid therapy, malnutrition etc.) or with a chronic lung disease and the non-invasive forms of Aspergillus lung diseases like aspergilloma (a fungus ball, composed of fungal hyphae, inflammatory cells, fibrin, mucus and tissue debris) develops in a pre-existing cavity within the lung parenchyma and allergic bronchopulmonary aspergillosis (ABPA) is a hypersensitivity reaction to Aspergillus antigens, occuring in the lungs that almost always affects patients with atopy, asthma or COPD and $15 \%$ of patients with cystic fibrosis 1 , $11,15,16,17,18$

Many of the above mentioned classes of fungal disorders are further sub-classified based on certain radiological findings as it is seen with aspergilloma, which is further divided into 3 forms(1) Chronic cavitary pulmonary aspergillosis, characterized by the formation and expansion of multiple cavities with the presence of fungal ball in some of the cavities, (2) Chronic fibrosing pulmonary aspergillosis characterized by pleural involvement in some cases expressed either as direct invasion of the pleural cavity or as fibrosis; thus though aspergilloma was initially categorized as non-invasive type yet the second form has shown considerable invasion, revealing a new site of it, (3) The third type is characterized by progressive enlargement of a single thin-walled cavity, which can take a turn towards CNA or show characteristics of CNA ${ }^{19}$.

Another form of Aspergillus infection has an expanded domain from chronic noninvasive or invasive indolent disease to acute necrotizing invasive disease occurring mainly in an immune suppressed patient, as can be seen with sinonasal fungal rhinosinusitis ${ }^{20}$. Other types of Aspergillus sinusitis are found to occur in immunocompetent young adults leading to bone destruction from erosion in $30-50 \%$ of cases, especially in the cribriform plate, posterior wall of the frontal sinus, ethmoid septa, lamina papyracea and medial antral wall ${ }^{21}$. The incidence of IPA is increasing day by day and has become the major fungal invasion of all mycoses as confirmed by the autopsy studies. The mortality rate of IPA exceeds $50 \%$ in neutropenic patients and reaches $90 \%$ in hematopoietic stem-cell transplantation (HSCT) recipients. A steady increase in the documented cases of IPA has been observed following HSCT, 
where the risk is much higher following allogeneic rather than autologous HSCT ${ }^{15,22,23,24}$.

It is reported that the incidence of invasive aspergillosis was between $5 \%$ to $10 \%$ in the case of allogeneic HSCT whereas for autologous HSCT it was less than $5 \%{ }^{13}$. Apart from invading the lungs infection may also disseminate haematogenously to other organs including brain which may lead to seizures, ring-enhancing lesions, cerebral infarctions, intracranial hemorrhage, meningitis and epidural abscesses. Other organs which may be less frequently involved are skin, kidneys, pleura, heart, esophagus, gastrointestinal tract, thyroid, spleen, and liver. IPA is the root cause of disseminated aspergillosis, and it was reported that $60 \%$ of the patients with IPA develops disseminated aspergillosis $1,15,25$.

Though Aspergillus skin infection as a primary lesion is extremely uncommon but Robert $\mathrm{P}$. Langlois et al, reported the first case where aspergillosis of the skin appeared as the primary lesion in a renal transplant recipient. They also stated about 10 cases of primary skin infection due to five different Aspergillus species; namely Aspergillus fumigatus, Aspergillus niger, Aspergillus flavus, Aspergillus glaucus and Aspergillus terreus ${ }^{26}$. Among all these species Aspergillus niger and Aspergillus flavus are of special mention in case of another similar type of invasion called "isolated sinus infection" were clinical and radiographic pulmonary findings were negative for Aspergillus but invasion of the sinus was proved by biopsy specimens and culture ${ }^{27}$. Depending upon the site of occurrence and the nature of the infection whether invasive or noninvasive, specific terminologies and further classification had been proposed as can be seen with David W Denning et al., who coined the term "Aspergillus tracheobronchitis" (AT) to describe bronchial and/or tracheal inflammation in patient with excess mucus production where Aspergillus is the only pathogen involved but without any invasion of bronchial mucosa as confirmed by biopsy studies.

Denning further classified AT into three types- (1) "Ulcerative Aspergillus tracheobronchitis" indicating histological invasion of the abnormal area of bronchial mucosa and/or cartilage showing hyphae consistent with Aspergillus and usually found at the suture line in lung transplantation recipients (2) "Pseudomembranous Aspergillus tracheobronchitis" indicating patients with extensive involvement of the whole of the tracheobronchial tree with a membranous slough overlying the mucosa containing Aspergillus and is the most severe form usually presents with cough and dyspnoea and the last type termed as (3) "Obstructing bronchial aspergillosis" is a condition characterized by the presence of thick mucous plugs full of Aspergillus in the airways with little or no inflammation and no evidence of invasion or allergic manifestations ${ }^{15,28}$.

All the above mentioned fungal disorders have a strong correlation with the immune status of the patient and are opportunistic in nature but there is another documentation in favor of primary aspergillosis of the larynx; a case study of a relatively healthy 73-year-old female patient suggested that nebulized tobramycin had been more hazardous than oral administration of antibiotics and claimed that both corticosteroid therapy and nebulized tobramycin were risk factors in the development of primary aspergillosis of larynx. This fungus was reported to show sign and symptoms mimicking precancerous conditionleukoplakia (a white patch lesion of the mucous membrane) of larynx; ${ }^{29}$ another case report of a 34year-old male patient shows that Aspergillus can also attack the immune-competent individual, intruding apex of the orbit causing proptosis of eye and chronic headache ${ }^{30}$.

Thus, a proper diagnosis is a prerequisite to differentiate between neoplasia and mycosis for the initiation of the appropriate therapy. But designing a therapy regimen needs a sound knowledge of epidemiological and clinical facts and figures, for this purpose some authors define the patient population into three categories- proven, probable and possible type. Proven category for invasive pulmonary fungal infection should reflect the presence of fungal infiltrates with fungal pneumonia as confirmed by chest radiography and Chest computed tomography (CT scan); additionally the cultures of the samples obtained by thoracic needle aspiration, bronchoalveolar lavage or biopsy should identify Aspergillus species. 
Probable category patients had clinical evidence of pneumonia; chest X-ray and CT scan should reveal characteristics like halo sign, air-crescent sign, nodules, wedge-shaped or cavitating lesions, the cavity within the area of consolidation and bronchoalveolar lavage should be negative for other agents to cause pneumonia. Other minor symptoms which may be present are a cough, chest pain, hemoptysis, dyspnoea and physical finding of pleural rub. Patients with possible invasive fungal infection had shown persistent fever and neutropenia, and they also had pulmonary infiltrates or sinus opacifications. These definitions are mainly used in the context of clinical and epidemiological research, but not for clinical decision making ${ }^{11,31,32}$. Later a modified version of the patient classification was released by the European Organization for Research and Treatment. As per which the category of proven invasive fungal disease can apply to any patient, regardless of whether the patient is immunocompromised, whereas the probable and possible categories are proposed for immunocompromised patient's only ${ }^{33}$.
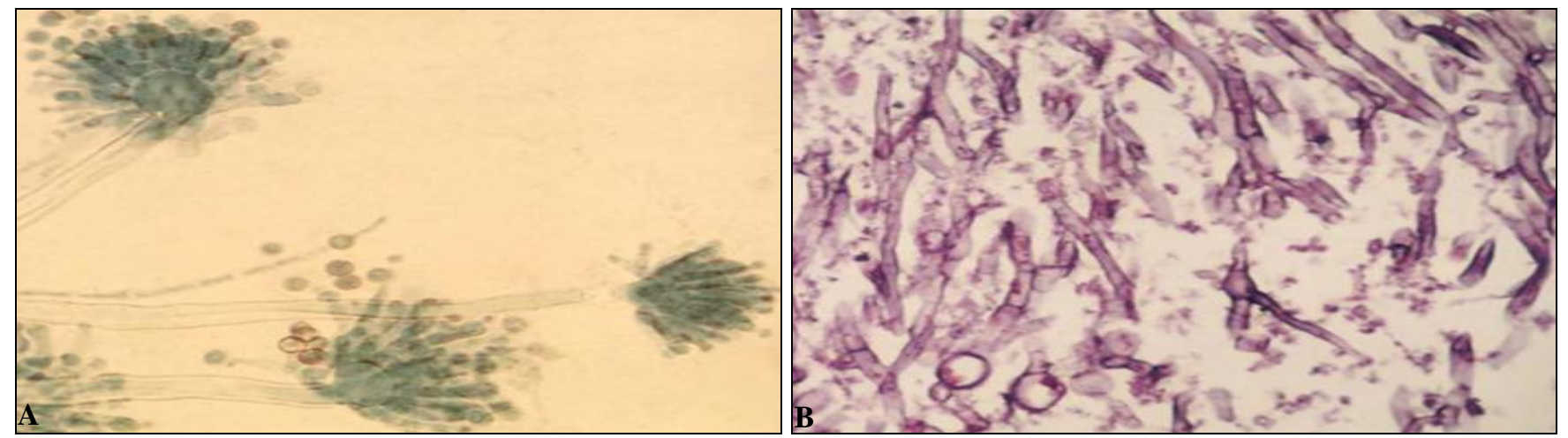

FIG. 1: MICROSCOPIC FEATURES OF ASPERGILLUS FUMIGATUS. (A) HIGH-POWER PHOTOMICROGRAPH DEMONSTRATES CONIDIOPHORES WITH THE CHARACTERISTIC HEAD APPEARANCE AND MINUTE SPORES. (B) MEDIUM-POWER PHOTOMICROGRAPH SHOWS SEPTATE HYPHAE BRANCHING AT AN ANGLE OF APPROXIMATELY 45 ${ }^{\circ}$. (TAKEN FROM EDUCATION EXHIBIT) ${ }^{34}$

Susceptibility to Aspergillus infection: Threequarters of invasive fungal infections in HSCT recipients are due to Aspergillus. Though lung has a strong defense mechanism against the inhaled Aspergillus conidia, mediated through receptorsToll-like receptors, dectin-1 and mannose-binding lectin, which identify specific fungal wall component and activate defence mechanism against Aspergillus hyphae by provoking cytokine release which in turn leads to neutrophil recruitment, yet susceptibility to fungal invasion increases because of certain therapeutic agent-induced suppression of the defence mechanism.

The susceptibility to this invasive infection increases with certain risk factors like graft versus host disease, prolonged neutropenia in the preengraftment phase of HSCT, severity and duration of immunosuppressant therapy, high doses and duration of corticosteroid therapy ( $>3$ weeks), use of broad-spectrum antibiotics, use of intravenous catheter, parenteral nutrition, renal failure, iron overload and cytomegalovirus (CMV) infection ${ }^{6}$, $11,15,35,36,37$
CMV facilitates bacterial and fungal infection in organ transplant recipients and may increase their mortality rate ${ }^{10}$. A study of patients with acute leukemia was done to identify significant risk factors for IPA and it was found that the most important risk factor was neutropenia, with a neutrophil count $<500$ cells $/ \mathrm{mm}^{3}$.

Early in the course of granulocytopenia patients developed signs of IPA at a rate of approximately $1 \%$ per day. The rate increased with the duration of granulocytopenia; approximating $4.3 \%$ per day between the 24th and 36th days ${ }^{38}$. Duration and severity of cytotoxic chemotherapy and duration of myelosuppression are among the other factors which contribute to IPA ${ }^{6}$. Incidence of IPA in AIDS patient is generally uncommon due to the administration of antiretroviral therapy but not in advanced or full-blown AIDS; still many cases were registered, almost all of which were associated with a low CD4 cell count $(<100$ cells $/ \mathrm{mm}^{3}$ ) and half of the HIV infected patients in the study group had coexistent neutropenia or were on corticosteroid therapy ${ }^{11,15}$. 
Susceptibility to IPA in COPD patient is considered to be favored by the state of progression of the disease itself (advanced COPD) and the disease associated with corticosteroid therapy. Other factors favoring IPA are structural changes in lung architecture, broad-spectrum antibiotic treatment, invasive procedures, mucosal lesions, impaired mucociliary clearance, comorbid illnesses such as diabetes mellitus, alcoholism, and malnutrition. COPD patients are also susceptible to CNA ${ }^{11,15}$. Alcohol intoxication has been linked with increased susceptibility to IPA as reports suggest that alcohol has a suppressive role ongranulocyte migration, bacterial clearance from lungs by macrophages and defective bactericidal activity by granulocytes ${ }^{39}$.

Symptoms of Aspergillus Infection: Symptoms of IPA mimic bronchopneumonia presented with fever unresponsive to antibiotics, cough, sputum production, dyspnoea, pleuritic chest pain and haemoptysis (haemoptysis occurs as a result of the invasion of blood vessels of the lung). Severe cases of invasive aspergillosis have additional symptoms like the swollen eye on one side, difficulty in talking, paralysis of facial muscles, the occurrence of ulcers inside the mouth or inside the chest wall and confusion, seizures or stroke-like symptoms indicating the spread of the fungal species to the brain. Some of these symptoms like fever, cough, and hemoptysis are common for CNA also; other symptoms present are malaise, fatigue, and weight loss. In certain cases, CNA may be asymptomatic whereas aspergilloma is mostly asymptomatic. The only prominent symptom for aspergilloma is mild hemoptysis.

Severe hemoptysis also occurs if associated with tuberculosis. The symptoms of ABPA include clinical asthma in almost all patients, but symptoms do not improve with the regular treatment of asthma and patients usually have episodic wheezing, expectoration of sputum containing brown plugs, pleuritic chest pain, shortness of breath and fever $11,14,15,17,40$. Symptoms of AT are a cough, fever, dyspnea (each present in less than $50 \%$ of patients), chest pain and hemoptysis; patients with pseudomembranous AT may develop unilateral monophonic wheeze or stridor which is as a result of obstruction of the airway with necrotic or fungal material.
The common symptoms of acute invasive Aspergillus rhinosinusitis include fever, cough, epistaxis and headache. Other symptoms which may be present occasionally are nasal discharge, sinus pain and sore throat. Symptoms of chronic invasive Aspergillus sinusitis which appear over months include diplopia, unilateral blindness, pain in the eye, proptosis, headache, loss or impairment of smell and nasal stuffiness ${ }^{2}$.

\section{Diagnosis: \\ Diagnosis of IPA:}

Sputum Test: Isolation of an Aspergillus species from sputum is highly predictive $(80 \%-90 \%)$ of invasive disease in immunocompromised patients, patients with leukemia and HSCT recipients, yet not a completely reliable method as $70 \%$ of patients with confirmed IPA were presented with negative sputum test. Moreover, the positive sputum test is not always a predictive marker of IPA as seen in a case study involving immunocompetent patients 11 , 15 .

Blood Culture: Many confirmed IPA patients have rarely shown a positive blood culture report. Moreover, it remains unknown how early these assays become positive in IPA, which becomes the most important problem in managing these patients. Even culturing of body fluids has a low diagnostic yield and does not always discriminate between invasive disease, colonization and contamination $11,15,41,42$.

Many tests were designed to detect circulating Aspergillus or fungal antigens for diagnosing IPA the latex agglutination test (LA), enzyme-linked immunosorbent assay (ELISA) and measurement of plasma $(1 \rightarrow 3) \quad-\beta$ - D-Glucan (BDG) concentration.

LA Test: In this test serum samples were serially diluted then; a diluted serum sample was mixed with IgG-coated latex on a clean glass slide. The mixture was incubated. Agglutination was observed and compared with a standard serum. Presence of clumps indicates a positive result, i.e. suspected particle is present and absence of clumps indicates a negative result, i.e. suspected particle is absent ${ }^{41,43}$.

ELISA Test: Although ELISA has a higher sensitivity than the LA test, yet it is not approved for diagnostic use in a certain country like Japan ${ }^{41}$. 
The LA test and ELISA detect circulating galactomannan antigens and are commercially available.

Luminex x-MAP Technology: This is a real-time and multiplex PCR-based technology that works on the same principle of sandwich bioassay like ELISA test. This test can overcome the limitations of ELISA by reducing sample volume, non-specific binding and cost etc. ${ }^{44}$ This technology is claimed to be rapid and reliable to identify fungal species and may be instrumental in routine clinical $\operatorname{diagnosis}^{45}$.

Galactomannan Assay: Galactomannan is a polysaccharide released by Aspergillus during hyphal growth and its presence in the serum can be detected by double-sandwich ELISA test, the best method to detect Aspergillus antigens several days before the presence of clinical signs, chest radiographic abnormalities or a positive culture. This assay employs the rat monoclonal antibody EB-A2 and recognizes the $1 \rightarrow 5-\beta$ - D-galactofuranoside side chains of the galactomannan molecule but the limitation of this assay is the inability to pinpoint the species involved. But incorporating galactomannan and quantitative PCR assays enhanced bronchoscopic identification of Aspergillus species particularly in bronchoalveolar lavage fluid in HSCT recipients. This assay is also a good tool for the diagnosis of cerebrospinal fluid for aspergillosis 11, 15, 42, 46 .

Other samples which can be used to conduct this test are a serum and pleural fluid ${ }^{47}$. A good reason for combining galactomannan and PCR assays might be the case where PCR identified IPA in one patient in a study population which was not diagnosed by galactomannan assay ${ }^{48}$. But if it is the case of acute pulmonary aspergillosis then it was reported that serum galactomannan test is less sensitive in non-neutropenic than in neutropenic patients, and hence the sample of choice for the non-neutropenic patient should be BAL fluid but not blood ${ }^{49}$. BAL fluid should be collected before initiating antifungal therapy as galactomannan becomes negative in the sample once antifungal therapy is on ${ }^{50}$.

Sometimes depending upon the antibiotics used earlier to the test one may get false positive results especially when the antibiotic is of fungal origin like penicillin or if EB-A2 has any cross-reactivity with the antibiotics as can be seen with piperacillin and tazobactam used as empirical antibacterial therapy in recipients of bone marrow transplantation ${ }^{51}$. A study by Christopher D. Pfeiffer et al., concluded that this test was more useful in patients who had a haematological malignancy or who had undergone hematopoietic cell transplantation than in solid-organ transplant recipients ${ }^{52}$.

\section{Measurement of Plasma BDG Concentration:} BDG is a ubiquitous component of fungi and determination of its plasma concentration is another useful screening method detecting deep mycoses including IPA ${ }^{11,41}$. But it is advisable to perform this test before the initiation of antifungal therapy especially with drugs like echinocandins, as they inhibit the biosynthesis of $(1 \rightarrow 3)-\beta$-D-Glucan in the fungal cell wall and may interfere with test result ${ }^{53}$.

This test is known to give false positive results in a patient population with no fungal invasion but with bacteraemia due to Alcaligenes faecalis or Streptococcus pneumoniae or Pseudomonas aeuroginosa, as these bacteria are also known to produce $\beta$-D-Glucan ${ }^{47,54}$.

Bronchoscopy: Bronchoscopy with bronchoalveolar lavage is generally helpful in the diagnosis of IPA especially in patients with diffuse lung involvement. Though the diagnostic yield of this method in the diagnosis of IPA is not consistent still it is a safe and useful tool in high-risk patients suspected to have IPA 5, 11, 15 . The certain study recommends the use of fiberoptic bronchoscopy in HSCT patients 55 .

\section{Biopsy with Histology: Histopathological} examination of lung tissue obtained by thoracoscopic or open-lung biopsy is considered the gold standard for the diagnosis of IPA. Presence of septate, branching hyphae in the lung parenchyma and obtaining a culture positive for Aspergillus using the same specimen are confirmative tests. The characteristic findings of biopsy vary depending upon the population of patient as can be seen with neutrophilic versus neutropenic patient group. 
The histopathological examination also allows for the exclusion of other diagnoses such as for malignancy and non-fungal infectious diseases 11 , 15. The however critical condition of the patient and thrombocytopenia remain as the limiting factors to carry out biopsy studies ${ }^{50}$.

Laser Capture Microdissection: This technique enables the collection of specific cells of interest with the help of an inverted light microscope fitted with a laser device to facilitate the visualization and procurement of cells and the sample thus collected can be subjected to PCR, electron microscopy, cell culture, and many other technologies. Though it has identified the single hypha of Aspergillus fumigatus still this technique has certain limitations and one of which may restrict its use to identify pauciseptate Mucormycete organisms ${ }^{56,57 .}$

Radiography: Although chest X-ray is a useful method for detecting IPA yet the findings are usually non-specific and findings indicative of IPA are often absent. Usual findings of radiograph include rounded densities, pleural-based infiltratessuggestive of pulmonary infarctions and cavitations $^{11,41}$.

Chest Tomography: Chest computed tomography (CT scan) combined with high-resolution images (HRCT) is a very useful technique employed routinely for early diagnosis, prevention and also to support the findings of other techniques like bronchoscopy and open-lung biopsy. Presence of nodules in the scan gives a clue to maximum chances to IPA. The most characteristic CT finding in cases of IPA is a halo sign, mainly seen in neutropenic patients early in the course of infection which corresponds pathologically to hemorrhage around a focus of pulmonary infarction or nodule. CT scan finds the exact location of the lesions and makes the selection of sampling site an easy task ${ }^{11 \text {, }}$ $15,41,58,59$

But if the patient is a neutropenic one the clinical signs and symptoms and changes in the results of imaging studies sometimes put the clinician in dilemma to decide whether to continue or abort the antifungal treatment approach, as the presence of cavitation in lung nodule, hemoptysis and air crescent sign in neutropenic patient does not necessarily indicate worsening of the fungal invasion or response to the antifungal therapy rather indicative of neutrophil recovery. It has also been reported that the average lesion volume in the lung can increase four times during the first week of effective antifungal therapy ${ }^{60}$. Thus, keeping track of neutrophil count may help to conclude.

Lateral Flow Test: This is a newer, faster and simpler technique to detect glycoprotein secreted by Aspergillus using monoclonal antibody JF5 in a lateral flow device. This technique acts on the principle of antigen-antibody reaction where the glycoprotein antigen secreted by the growing fungus binds with the monoclonal antibody. This technique can detect a glycoprotein antigen in the sera and BAL fluid of patients with invasive aspergillosis within $15 \min ^{47,59}$

Diagnosis of CNA: Diagnosis of CNA involves a clinical examination, imaging studies- chest radiograph and chest $\mathrm{CT}$ scan, the culture of sputum, bronchoscopy and serological tests.

Clinical Observation: Clinical examination considers chronic pulmonary or systemic symptoms. The duration of the prevailing symptoms like a productive cough, weight loss, and hemoptysis to be present for more than one month without the existence of any immunecompromising conditions (e.g., hematological malignancy, neutropenia, organ transplantation) and dissemination ${ }^{11,15}$.

Laboratory Diagnosis: Laboratory findings depend on elevated levels of inflammatory markers like C-reactive protein, plasma viscosity studies or erythrocyte sedimentation rate. Majority of patients are diagnosed with high levels of IgG antibodies. It also includes appropriate cultures and serological tests to rule out the involvement of other pathogens that are associated with similar disease presentation $^{11,15}$.

Histological Study: As the features of CNA are necrosis of lung tissue, acute or chronic inflammation of the cavity wall and presence of hyphae hence histological examination is of unquestionable value ${ }^{11,15}$.

Radiology: Radiological studies reveal that the upper lobes of the lungs are the common site of involvement in CNA and consider certain diagnostic characters like cavitary pulmonary 
lesions with para cavitary infiltrate, new cavity formation and expansion of the cavity size to confirm CNA. Imaging studies such as chest radiograph and chest $\mathrm{CT}$ scan usually show consolidation, pleural thickening and cavitary lesions in the lobes of upper lung and nearly 50\% of the patients may have aspergilloma ${ }^{11,15,19}$.

Diagnosis of Aspergilloma: Clinical examination, radiography, chest CT scan, and serological tests are performed.

Sputum Cultures: Sputum cultures for Aspergillus species are positive only in $50 \%$ of cases ${ }^{11,15}$.

Bronchoscopy: Bronchoscopy with bronchoalveolar lavage has shown the presence of Aspergillus antigen ${ }^{11,15}$.

Serological test: Most cases show the presence of IgG antibodies to Aspergillus but the test result may be negative if the patient is on corticosteroid therapy ${ }^{11,15}$.

Chest Radiography: It demonstrates the presence of a upper-lobe, mobile, intra-cavitary mass with an air crescent in the periphery ${ }^{11,15}$.

Chest Tomography: As the radiological appearances may be seen in other conditions such as neoplasm, abscess, hydatid cyst and granulomatosis with polyangiitis (Wegener's granulomatosis) hence CT scan is sometimes required especially when the results of chest radiography is not prominent ${ }^{11,15}$.

\section{Diagnosis of ABPA:}

Clinical Observation: It includes a screening of patients for clinical asthma, presence of episodic wheezing, expectoration of sputum containing brown plugs, pleuritic chest pain and fever ${ }^{11,15}$.

Aspergillus Skin Test: Patients once screened with clinical asthma are subjected to this test using $A$. fumigatus antigen, either by a skin-prick test or intradermal injection. Readings are taken every 15 min for one $\mathrm{h}$ and then after 6-8 $\mathrm{h}$. The reactions are further graded based upon the appearance of symptoms like wheal, erythema and edema ${ }^{16}$.

Serological Tests: It considers an elevated level of total serum IgE usually more than $1000 \mathrm{ng} / \mathrm{ml}$, serum precipitins of Aspergillus and peripheral eosinophilia with a cell count of 1000 cells/ $\mu$; histologic examination reveals the presence of mucus, fibrin, curschmann spirals, Charcot-Leyden crystals, inflammatory cells primarily the eosinophils and presence of hyphae ${ }^{11,15,16}$.

Radiology: Radiology and serological tests are the confirmatory tests. Though in the early stage of the disease radiography may not be helpful but during acute exacerbations it shows fleeting pulmonary infiltrates (characteristic feature) which tend to appear in the upper lobe and are central in location, presence of band-like opacities flowing out from the hilum as a result of mucoid impaction of the airways, other radiological sign present is thickened and inflamed bronchi ${ }^{11,15,61}$.

Chest Tomography with High Resolution: HRCT is the better technique to demonstrate the characteristic features like mucoid impaction, centrilobular nodules, atelectasis, bronchial wall thickening and the later changes of ABPA in the form of central bronchiectasis and pulmonary fibrosis $11,15,62$.

Diagnosis of Acute Invasive Fungal Rhinosinusitis: Fungal culture: It was found that fungal cultures were negative in almost $30 \%$ of the times. The slow growth rate of certain fungal species is another hindrance especially when the treatment has to start in no time ${ }^{20}$.

In-situ Hybridization Method: In this method, rRNA sequences have been used as optimal targets for in situ hybridization. As rRNA molecules are a useful tool for phylogenetic classification of fungi owing to their abundance and species specificity hence this method may be useful in the identification of species in specimens with negative culture ${ }^{20}$.

Antifungal Prophylaxis / Treatment: Analysis made in the year 2008 by D. Neofytos et al., based on Multicenter Prospective Antifungal Therapy (PATH) Alliance Registry had shown that 6-week survival rate has significantly improved among HSCT recipients with invasive aspergillosis as compared with the previously reported data; this reflects a better understanding of the epidemiology of the invasive fungal infection ${ }^{63}$. The survival of HSCT recipients depends on early or timely diagnosis and treatment with antifungal agents. 
Y. Hicheri et al., reviewed the prophylactic use of second-generation broad-spectrum antifungal voriconazole to reduce the mortality from invasive fungal infection in such recipients. Similar efficacy and tolerability have been seen with an antifungal antibiotic like liposomal amphotericin B for the treatment of breakthrough invasive aspergillosis in patients under azole prophylaxis; except for inhaled liposomal amphotericin B which is effective only in case of invasive pulmonary aspergillosis in patients concomitantly treated with fluconazole.

A study conducted by Bart J. Rijnders et al., on adult patients with hematologic disease concluded that when aerosolized liposomal amphotericin B was used prophylactically it significantly reduced the incidence of IPA, a total dose of $2.5 \mathrm{ml}$ of a 5 $\mathrm{mg} / \mathrm{ml}$ solution was used for nebulization (duration of each nebulization $1 / 2 \mathrm{~h}$ ) where nebulization was performed for two consecutive days per week and weekly treatment was continued till neutropenia resolved. A maximum of 12 inhalations per neutropenic episode was given. The reason behind accepting voriconazole as a prophylactic agent for invasive aspergillosis is its good tissue penetration in the lungs (the primary site of infection in invasive aspergillosis) following oral administration. Though voriconazole is suitable for prolonged therapy because of its better tolerability and milder side effects when compared with amphotericin B yet it is associated with the chances of certain potential adverse effects in seriously ill patients- prolonged visual disturbances, QTinterval prolongation, hallucinations, photosensitivity, hepatic toxicity etc. and it is also associated with a significant number of drug-drug interactions, such as with cyclosporine, warfarin, terfenadine, carbamazepine, quinidine, rifampin, statins and sulfonylureas 6,11,15, 64, 65, 66 .

Thus should be cautiously used in patients with neuropathy, arrhythmia and hepatic disorders with proper monitoring of conditions. As fluconazole had already been a well-established drug in the market before voriconazole was introduced, hence a comparative study of the efficacy profile of both the drugs became inevitable; Y. Hicheri et al., in their comparative study between voriconazole and fluconazole for 6 months and extended till 12 months showed similar efficacy for reducing the mortality in graft recipients but exploratory analyses had shown that voriconazole might have significantly improved 6-month fungal-free survival $(78 \%$ versus $61 \%, \mathrm{p} 0.04)$ and reduced incidence of invasive fungal infections $(9 \%$ versus $21 \%, \mathrm{p}$ 0.04) when compared with fluconazole in the higher-risk subpopulation of patients with acute myeloid leukemia. Another trial shows that voriconazole has higher prophylactic success when compared with itraconazole. Voriconazole is claimed to be the better prophylactic agent because of its better long-term tolerability especially in case of invasive aspergillosis which can occur up to six months after transplantation ${ }^{6}$.

Another study reported the next year (2013) by Petros Pechlivanoglou et al., on HSCT patient claimed that the risk of invasive fungal infection after prophylactic use of voriconazole or posaconazole was lesser than after fluconazole or itraconazole usage; the study reported posaconazole as the most effective prophylactic agent in neutropenic patients ${ }^{67}$. Itraconazole is recommended for patients with stable disease who are still receiving chemotherapy for leukemia ${ }^{68}$. Drug used and approved by the USFDA as prophylaxis for invasive Aspergillus infections in neutropenic patients and in patients aged $\geq 13$ years is posaconazole because of its better tolerance; the oral suspension of posaconazole needs to be administered with food or a nutritional supplement to assure adequate bioavailability and as it is mainly eliminated in the feces as unchanged drug and renal clearance has almost negligible role hence dose adjustment is not required in case of renal or hepatic insufficiency ${ }^{69}$.

The recommended dose of voriconazole in IPA is 6 $\mathrm{mg} / \mathrm{kg}$ twice daily intravenously on day 1 , followed by $4 \mathrm{mg} / \mathrm{kg}$ /day. After 7 days switching to $200 \mathrm{mg}$ p.o. twice daily 15,70 , but a prior creatinine clearance test to be performed before the intravenous therapy is started as the intravenous formulation is contraindicated in patients with creatinine clearance rate less than $50 \mathrm{ml} / \mathrm{min}^{69}$. When it comes to the duration of the treatment of IPA, Infectious Diseases Society of America (IDSA) suggests minimum treatment duration of 612 weeks to be considered whereas immunosuppressed patients should be treated throughout the period of immune suppression and continue until lesions have resolved. As per IDSA guideline 
voriconazole is the primary drug for IPA but alternative drugs which can be used are liposomal amphotericin B at 3-5 mg/kg/day iv, amphotericin B lipoidal complex at $5 \mathrm{mg} / \mathrm{kg} /$ day iv, caspofungin at $70 \mathrm{mg}$ on day1 followed by $50 \mathrm{mg} /$ day by iv route on subsequent administration ${ }^{70}$.

Another drug which can be used as alternative to voriconazole is is a vuconazole as a review published on 2015 by Marisa H. Miceli et al., reports that a newer USFDA approved drug is a vuconazole has efficacy non-inferior to voriconazole to treat invasive aspergillosis and also this drug is well tolerated both by patients and normal volunteers with few serious adverse effects and fewer drug-drug interactions as compared to voriconazole. Side effects of voriconazole like visual disturbances, hallucinations and photosensitivity have not been described with is a vuconazole ${ }^{65}$. Though voriconazole is the primary drug a for IPA, a study conducted by Kieren A. Marr et al., had shown that combination of voriconazole and caspofungin for salvage therapy of invasive aspergillosis not only improved 3month fungal-free survival rate but also reduced the mortality rate as compared to the therapy with voriconazole alone ${ }^{71}$.

Another study conducted by Oliver A. Cornely et al., advocated for the use of liposomal amphotericin B as the first line drug for the treatment of IPA in highly immunocompromised patients 72 . Among, all the formulations of amphotericin B, lipoidal and liposomal amphotericin B have a special role to treat IPA in patient with impaired renal function or not tolerating the deoxycholate amphotericin B and developing nephrotoxicity ${ }^{21,53,71}$.

But a study by Oliver A. Cornely et al., had shown that even liposomal amphotericin B produces nephrotoxicity and hypokalemia when administered at a dose as high as $10 \mathrm{mg} / \mathrm{kg}$ per day moreover no additional benefit was observed with this dose as compared to another study group receiving $3 \mathrm{mg} / \mathrm{kg}$ daily dose. In this study of Oliver A. Cornely et al., the patient population was divided into two groups where one group received $3 \mathrm{mg} / \mathrm{kg} / \mathrm{day}$ and other group received $10 \mathrm{mg} / \mathrm{kg} / \mathrm{day}$ of liposomal amphotericin B for 14 days following which all patients were treated with $3 \mathrm{mg} / \mathrm{kg} /$ day until the end of the study and the conclusion was drawn after 12 weeks of observation ${ }^{72}$.

But another study published by Thomas J. Walsh et $a l$., much earlier to the study of Oliver A. Cornely et al., claims that liposomal amphotericin B at a dose as high as $15 \mathrm{mg} / \mathrm{kg} /$ day was well tolerated, concluded based on 7 days observation of the evaluable patient population; the study also suggests that if invasion of the fungus continues at different sites even when the therapy is on with a dose of $10 \mathrm{mg} / \mathrm{kg} /$ day then the dose can be increased to $15 \mathrm{mg} / \mathrm{kg} /$ day under rational therapeutic approach ${ }^{31}$. A report submitted by $\mathrm{T}$. Yeghen et al., recommends the use of combination of amphotericin B or a lipid-based formulation plus GM-CSF $(5 \mathrm{mg} / \mathrm{kg} /$ day) on patients showing at least one lesion with imaging studies suggestive of aspergillosis, whereas surgery is reserved for patients with worsening lesion 22 .

David W. Denning et al., suggested that in case of azole resistant Aspergillus fumigatus treatment of chronic pulmonary aspergillosis can be done using either liposomal amphotericin B thrice weekly or six times weekly therapy with micafungin or caspofungin through a Port-A-Cath ${ }^{73}$. Neutropenic and immunosuppressed patients in allogenic transplantation population are treated with voriconazole as secondary prophylaxis to prevent fungal relapse, voriconazole showed promising result as secondary prophylaxis at $400 \mathrm{mg} /$ day (intravenously or orally for 44 to 245 days) where relapse of invasive infection had taken place.

Voriconazole was also found suitable in leukaemia patients as there was no reported significant adverse drug reaction when concomitant therapy for acute leukaemia was on but dose adjustment is required in certain cases to reduce the toxicity due to concomitantly administered drugs. Other agents which have shown efficacy in the treatment of IPA in patients who cannot tolerate the first line agents or in case of refractory IPA are echinocandin derivatives such as caspofungin, micafungin and anidulafungin. There is good evidence favouring the use of caspofungin in salvage therapy $6,15,53,74$ 75 . Further, a case study has reported that fungal strain like Aspergillus ustus was found resistant to multiple antifungal medications and had shown sensitivity only to caspofungin thus making it the 
drug of choice for the treatment of disseminated fungal infection of the hand, lung and thigh etc. ${ }^{9}$

A clinical study reported that echinocandins augment the efficacy of liposomal amphotericin $\mathrm{B}^{75}$ but another study conducted by Jill P. Adler-Moore et al., for the treatment of murine disseminated and pulmonary aspergillosis caused by Aspergillus fumigatus revealed that liposomal amphotericin B plus echinocandin or liposomal amphotericin B prior to echinocandin was as effective as liposomal amphotericin B alone ${ }^{76}$. Yet another study conducted by Jill P. Adler-Moore et al., on murine systemic Aspergillus flavus infections suggested that concomitant but not subsequent administration of liposomal amphotericin B and micafungin or caspofungin treated the infection effectively but when it comes to pulmonary Aspergillus flavus infection liposomal amphotericin B suppressed the infection effectively but not caspofungin ${ }^{77}$.

Selection of a proper prophylactic agent determines the success rate of a treatment as guided by the consensus of European guidelines on antifungal prophylaxis and IDSA grading system, which divide high-risk haematology patients into three groups for the purpose of assigning the most appropriate agent for primary antifungal prophylaxis. The first group includes patients receiving the induction chemotherapy for acute leukaemia, for which the strongly recommended agent is posaconazole followed by aerosolized liposomal amphotericin B in combination with oral fluconazole then comes intravenous (i.v) / oral fluconazole, oral itraconazole solution and intravenous polyenes. The second patient group consists of allogeneic haematopoietic stem cell transplant recipients during the neutropenic phase, for which the drug of choice is i.v/oral fluconazole or oral voriconazole (provisional at that point of time), next comes itraconazole (i.v then oral) followed by the combination of aerosolized liposomal amphotericin B and oral fluconazole, the last choice being i.v micafungin or i.v polyenes.

But a study reported by Thomas J. Walsh et al., claimed that echinocandin like caspofungin was as effective as liposomal amphotericin B but shown better tolerance than liposomal amphotericin B when given as empirical antifungal therapy. The third patient group includes allogeneic haematopoietic stem cell transplant recipients with graft-versus-host disease for which the drug of choice is oral posaconazole, oral voriconazole (the then provisional), next choice is itraconazole (i.v then oral), the third choice is oral/i.v fluconazole or i.v polyenes ${ }^{6,78}$. A comparative study by Andrew J. Ullmann et al., between posaconazole and fluconazole revealed that both these drugs have similar prophylactic efficacy but posaconazole was superior in preventing invasive aspergillosis and reducing the death rate in patients with graftversus-host disease ${ }^{79}$. Voriconazole also becomes the drug of choice because of its significantly better efficacy against Aspergillus terreus the second commonest species of Aspergillus (mainly responsible for nosocomial infections), to cause IPA in cancer patient and this species is likely to be resistant to amphotericin $\mathrm{B}$; another study reveals the potential of voriconazole to treat IPA due to Aspergillus ustus in a patient with acute myeloid leukemia and neutropenia, where wedge resection of the pulmonary lesion had been performed ${ }^{7,15,70}$.

As per IDSA guideline treatment of aspergillosis of the central nervous system is the same like that of IPA ${ }^{70}$. Baslar et al., in their case report of a 18 years girl, a HSCT recipient, has shown successful treatment of invasive aspergillosis of the central nervous system with the combination of liposomal amphotericin B and itraconazole, where liposomal amphotericin B was initially given at a dose of $1 \mathrm{mg} / \mathrm{kg} /$ day then increased to $2 \mathrm{mg} / \mathrm{kg} /$ day and continued for 3 months and then stopped with a cumulative dose of $6775 \mathrm{mg}$, whereas itraconazole was continued at a dose of 200-400 mg/day till 2 years for complete remission of the disease ${ }^{21,80}$.

In contrast to this report there is another case report by Prakash et al., which states that amphotericin B (either as deoxycholate or liposomal form) poorly penetrates across the blood-brain barrier leading to the treatment failure and death of a 14-year-old boy with acute lymphoblastic leukemia and multiple brain abscesses due to cerebral Aspergillus infection; prophylaxis with amphotericin B was initiated at $0.5 \mathrm{mg} / \mathrm{kg}$ on alternate days and after confirmation of diagnosis dose was increased to 1.5 $\mathrm{mg} / \mathrm{kg}$. Prakash et al., further stated that most antifungal agents are large molecules with molecular weight more than 700 Da which does not allow sufficient penetration of the antifungals into 
the brain or cerebrospinal fluid; amphotericin B (either as deoxycholate or liposomal form), echinocandins like caspofungin and itraconazole poorly penetrate across the blood-brain barrier whereas voriconazole has better penetration in the CNS with a cerebrospinal fluid to plasma ratio of 0.22 to $1^{81}$. Now, despite of poor penetration profile of certain drugs in the brain, the success of treatment of aspergillosis of CNS in the above mentioned case report by Baslar et al., may be justified by certain facts like severity/stage of the infection which may allow a long term therapy to go on with a low tissue/fluid concentration attained by the drug, duration of treatment and combination therapy with drugs where one drug may have a compensatory role to insufficient tissue penetration of the other. Aspergillus meningitis is treated with systemic and intrathecal amphotericin B or itraconazole and voriconazole, whereas moderate evidence suggests the need for surgical drainage along with systemic therapy for epidural abscesses 21 .

Response to therapy in AIDS patient group is poor with median survival of 3 months following diagnosis ${ }^{15}$. The treatment of invasive aspergillosis in pediatric patients with acquired immunodeficiency arising as an indirect consequence of either hematological cancer or allogeneic hematopoietic stem cell or solid organ transplantation, is largely dependent on age group; a report by Athanasios Tragiannidis et al., says that primary drugs to treat invasive aspergillosis in patients aged 2 years and above (up to 12 years) are voriconazole and liposomal amphotericin B, whereas the second-line drugs are amphotericin B lipid complex, amphotericin B colloidal dispersion and caspofungin.

If the patient is aged less than 2 years then the first line drug is none other than liposomal amphotericin $\mathrm{B}$ whereas the second-line treatment includes agents like amphotericin B lipid complex and caspofungin. Neonates can be treated with either amphotericin B deoxycholate or liposomal amphotericin B or amphotericin B lipid complex and patient with age group between 13-18 years can be treated with amphotericin B deoxycholate or liposomal amphotericin B or amphotericin B lipid complex or amphotericin B colloidal dispersion or voriconazole (oral suspension / capsule / i.v. solution) or posaconazole oral suspension or itraconazole (oral suspension/ capsule) or caspofungin ${ }^{82}$.

The therapy for CNA is similar to the treatment strategy of IPA, generally itraconazole is given daily at $400 \mathrm{mg}$ but if the treatment fails then amphotericin B deoxycholate is given up to a total dose of $2 \mathrm{~g}$; whereas refractory cases can be treated with a combination therapy of both and in case of clinical deterioration intracavitary instillation of amphotericin B was proposed ${ }^{14}$. Selection of drugs for the treatment of CNA depends on the severity of the disease. The primary agent for treatment of CNA is voriconazole especially in renal transplant patient and the alternative agent is itraconazole. Severe cases are treated with i.v voriconazole or liposomal amphotericin B, even surgical resection is also proposed.

A study shows that $80 \%$ of patients showed improvement when voriconazole was given at 200 mg twice daily for a period of 4-24 weeks as primary or salvage therapy. Amphotericin $\mathrm{B}$ given at a dose of $0.5-1 \mathrm{mg} / \mathrm{kg} /$ day also shown positive results. The dose for the lipid formulation of amphotericin B was 4-5 mg/kg/day 11, 14, 15 . For majority of cases the total dose of conventional amphotericin B (dry powder along with deoxycholate for extemporaneous dispersion) given is 3-4 g over 2-3 months. Starting dose is $0.3 \mathrm{mg} / \mathrm{kg}$ infused over 4-8 h but based on tolerance dose can be increased up to $0.7 \mathrm{mg} / \mathrm{kg}$; when it comes to liposomal amphotericin B a study confirms that monotherapy with $15 \mathrm{mg} / \mathrm{kg} /$ day was well tolerated 31, 83. Treatment strategy for aspergilloma depends on its types- chronic cavitary and single aspergilloma. Single aspergilloma does not need antifungal therapy but surgical therapy is required depending upon circumstances but chronic cavitary aspergilloma requires long-term therapy with itraconazole or voriconazole; the alternative treatment approach for aspergilloma is similar to the treatment of IPA ${ }^{70}$.

Antifungal treatment starts soon after the patient becomes symptomatic with hemoptysis. M. Kousha et al., in their review suggested that CT-guided percutaneous administration of amphotericin B could be effective for aspergilloma especially in patients with massive hemoptysis where resolution 
observed within few days, but the role of i.v. amphotericin B is uncertain. Administration of itraconazole in case of life-threatening aspergilloma was doubted due to its slow onset of action but if administered at 100-200 mg/day, a significant level of the drug within the aspergilloma cavity was demonstrated. Bronchial artery embolization is a temporary approach for lifethreatening hemoptysis; a study with six patients with hematologic malignancy has shown successful surgical resection of cavitating IPA, allowing a cytotoxic therapy to go on smoothly in patients with prolonged neutropenia.

Surgical resection is considered in case of recurrent hemoptysis or in case of IPA with prolonged neutropenia (more than ten days) provided pulmonary function tests permit the procedure. A study with fourteen cases of aspergilloma had shown a survival rate of $92.85 \%$ following surgical resection with no recurrence during an observation period from six months till ten years $4,11,15,84,85$.

Treatment of ABPA is based on the reduction of hypersensitivity and inflammatory phase of the disease; hence oral corticosteroids become the drug of choice for the treatment. Oral prednisone is generally given at a dose of $0.5 \mathrm{mg} / \mathrm{kg} /$ day for 2 weeks, followed by gradual tapering of the dose; use of pulse doses of i.v methylprednisolone for the treatment of severe ABPA is also documented. However, many patients needed a prolonged therapy with corticosteroids determined based on the serum IgE level but prolonged corticosteroid therapy induced adverse effects (growth retardation, adiposity, hypertension and osteoporosis) limited the use of corticosteroid, hence treatment was started with a subcutaneously administered single dose of $300 \mathrm{mg}$ of recombinant anti-IgE antibody (omalizumab) without any corticosteroids which resolved dyspnoea and improved the lung function test (forced expiratory volume) but a second dose was required after 2 weeks.

Treatment with itraconazole at $200 \mathrm{mg}$ twice daily for 16 weeks also helped to reduce the dose of corticosteroids to $50 \%$ and a reduction in serum IgE concentration (25\%) and either improvement of pulmonary function test results or partial to complete resolution of pulmonary infiltrates.
Therapy was also given to a single patient with amphotericin B and budesonide 15, 16, 17, 86 . Itraconazole is also preferred in patients not responding to oral corticosteroid or if a corticosteroid-sparing treatment approach is required ${ }^{87,88}$. But precaution should be taken while giving itraconazole capsule concomitantly with other drugs which show the pharmacokinetic type of interaction hence serum itraconazole level should be monitored to ascertain adequate absorption of the drug. In case of inadequate absorption cyclodextrin oral suspension of itraconazole is preferred over capsule because of superior absorption of the former ${ }^{21}$. Other alternative drugs used for ABPA are voriconazole (200mg p.o every $12 \mathrm{~h}$ ) or posaconazole (400 mg p.o twice daily) ${ }^{70}$. Successful treatment of invasive primary aspergillosis of larynx in a 73-year-old woman with oral voriconazole for five months makes this antifungal as the drug of choice for old patients ${ }^{29}$. But many cases of laryngeal infection had a crucial need for surgical debridement or excision in addition to systemic antifungal therapy 21.

The in-vitro study conducted by David A. Stevens et al., on Aspergillus fumigates had shown the efficacy of single as well as combination therapy with several agents in different assays like MTT [3(4,5-dimethylthiazol-2-yl)-2,5-diphenyltetrazolium bromide] and XTT [2,3-bis(2-methoxy-4-nitro-5sulfophenyl)-2H-tetrazolium-5-carboxanilide]. The different therapeutic agents taken by David A. Stevens et al., for the study are voriconazole, amphotericin B, polymorphonuclear neutrophils, monocyte, granulocyte colony-stimulating factor (G-CSF) and granulocyte-macrophage colonystimulating factor (GM-CSF). In both the assays the combination of neutrophil and voriconazole gave additive results towards inhibition of fungal hyphae; neutrophil alone has shown certain degree of hyphael inhibition when treated with G-CSF or GM-CSF whereas the combination of voriconazole and neutrophil-treated with G-CSF or GM-CSF has shown the most significant inhibition.

Combinations of voriconazole and monocyte or voriconazole and GM-CSF treated monocyte have also shown efficacy but the treatment of monocyte with GM-CSF did not show a significantly higher value of hyphael inhibition as compared to GM- 
CSF untreated monocyte. Combination of amphotericin B and neutrophil was also tried but did not show additive effect rather the efficacy level went down when compared with the monotherapy with amphotericin B ${ }^{89}$. In-vitro studies conducted by Cornelia Lass-Florl on Aspergillus terreus using several antifungals demonstrated that amphotericin B is not at all a good choice to treat because of resistance but anidulafungin, micafungin, posaconazole, and itraconazole have shown to be effective ${ }^{90}$. Both primary and alternative treatments of invasive sinus aspergillosis and tracheobronchial aspergillosis are similar to the treatment of IPA. Primary treatment of aspergillosis of heart (endocarditis, pericarditis and myocarditis), osteomyelitis, septic arthritis, cutaneous asper-gillosis, and aspergillus peritonitis was done with deoxycholate amphotericin B but voriconazole also proved its efficacy and was also considered as a primary antifungal agent for these patients; whereas the alternative treatments for all these remain the same like that of IPA.

Surgical resection is recommended for lesions of the endocardium and pericardium of heart, devitalized bone, cartilage and in case of cutaneous aspergillosis. As the concentration of amphotericin $\mathrm{B}$ attained in bone is less hence additional drugs like rifampin or flucytosine may be required; itraconazole may also be used as a promising candidate because it attains a higher concentration in bone. Aspergillosis of the eye (endophthalmitis and keratitis) is treated well with intraocular amphotericin B (dose $10 \mu \mathrm{g}$ ) and partial vitrectomy but voriconazole can also be used in place of amphotericin B. Keratitis to be treated topically. The alternative approach for aspergillosis of eye remains the same like that of IPA ${ }^{21,70}$.

Orbital invasive aspergillosis presented with a chronic headache, proptosis, CT scan representing heterogeneous, hyperdense mass infiltrating the orbital apex, optic neuritis and orbital bacterial cellulitis or orbital abscess cellulitis was treated well with i.v amphotericin B $0.8 \mathrm{mg} / \mathrm{kg} /$ day, for two weeks ${ }^{30}$. Moderate evidence suggests that Allergic Aspergillus sinusitis can be treated by surgical drainage with additional antibiotic therapy for secondary bacterial infection. Systemic therapy with itraconazole is reserved for definite evidence of tissue invasion or orbital/intracranial extension.
Another measure for symptomatic relief is use of nasal corticosteroids and saline douches, though systemic corticosteroids are indicated in cases refractory to surgery yet their use immediately after surgery should be avoided or reconsidered as postsurgical systemic corticosteroid therapy may interfere with the healing of the surgical wounds ${ }^{21,70}$. Aspergillus lymph node infection is treated with systemic antifungal therapy whereas refractory cases need for surgical resection ${ }^{21}$.

Success Rate of Treatment: The rate of success of the much-advocated azole therapy with standard drugs- itraconazole, voriconazole, and posaconazole, has come down due to widespread resistance to these antifungals as a result of unscrupulous and injudicious use of antifungals in cultivation. The highest prevalence of resistant isolates was found in cystic fibrosis patients. A mutation in Cyp51A protein, a central enzyme with lanosterol-14 $\alpha$-demethylase activity in the ergosterol biosynthesis pathway of fungi is associated with resistance. Several mutations involved with triazole resistance are- $\mathrm{L98H}$ with tandem repeat, M220I, and F219C. Fungi with G54W substitution have shown high resistance to both itraconazole and posaconazole whereas all other substitutions are associated only with itraconazole resistance. Apart from mutations, some other mechanisms are also suspected behind resistance as certain fungal isolates did not exhibit any such mutations but were resistant to itraconazole. Many itraconazole-resistant strains were found to show cross-resistance to voriconazole, and posaconazole with moderately increased minimum inhibitory concentration (MIC) values $^{73,91}$.

Another factor hampering success rate of the therapy is the limitation of diagnosis to discriminate between two different types of fungal invasions with overlapping symptoms. This can be seen in case of zygomycosis and aspergillosis where identifying these particular species by biopsy in cancer patients with thrombocytopenia is difficult and other radiological features differ very slightly in both the groups except for a difference in the number of nodules; initiation of therapy with voriconazole in patients with zygomycosis may lead to further spread of zygomycosis as voriconazole has no activity against zygomycosis 
${ }^{92}$. To solve this issue of overlapping identifying characters PCR technique can be used, as Volker Rickerts et al., concluded that PCR assays offered a reliable etiologic diagnosis that was superior to culture in patients with proven invasive mold infection and PCR assays successfully distinguished between aspergillosis and zygomycosis; further combination of PCR with culture method had increased the diagnostic yield from $63 \%$ to $96 \%$; this will help to increase the success rate of therapy ${ }^{93}$.

Other reasons behind the failure of therapy are a low concentration of drug at the infection site, drug interactions and certain host factors like-severity of illness and persistence of immunodeficiency ${ }^{94}$.

CONCLUSION: A timely and correct diagnosis of the invasion is the prime requisite for the success of a treatment. Histopathological diagnosis, culture, Galactomannan assay, PCR, etc. play a very important role in the identification of fungal species and initiation of fungal-specific therapy. It is better to run a routine creatinine clearance test to facilitate an early and safe therapy with intravenous voriconazole whenever fungal invasion is suspected; except for the case with zygomycosis where the use of voriconazole is ruled out.

If treatment to be initiated with amphotericin B and a high dose is considered then it is better to give high dose for seven days till which is was found safe and then reduce the dose to avoid any discrepancy regarding the maximum tolerated dose and the duration of the treatment, but irrespective of duration of treatment patients should be monitored for any sign of toxicity. It is better to choose or develop anti-fungals restricting the molecular weight to $\leq 700 \mathrm{Da}$ to treat fungal invasion of cerebrospinal fluid or brain, as compounds with higher molecular weight do not show adequate penetration leading to the failure of the therapy.

\section{ACKNOWLEDGEMENT: None}

\section{CONFLICT OF INTEREST: None}

\section{REFERENCES:}

1. Mehmood T, Matt JC, and Khasawneh FA: A 52-year-old HIV-positive man with abdominal pain.Canadian Journal of Infectious Diseases and Medical Microbiology 2015;
26(2): 97-9. Available from: downloads.hindawi.com/ journals/cjidmm/2015/849343.pdf

2. Denning DW: Invasive aspergillosis. Clinical Infectious Diseases 1998; 26: 781-803.

3. Rankin NE: Disseminated aspergillosis moniliasis associated with agranulocytosis and antibiotic therapy. British Medical Journal 1953; 91: 8-9.

4. Moreau P, Jean-Ralph Z, Noel M, Oliver B, Beatrice M, and Depei W: Localized invasive pulmonary aspergillosis in patients with neutropenia. Cancer 1993; 72(11): 3223-6. http://onlinelibrary.wiley.com/doi/10.1002/1097-0142 (19931201)72:11\%3C3223::AID-CNCR2820721115\%3E 3.0.CO;2-R/pdf

5. Reichenberger F, Habicht J, Matt P, Frei R, Soler M, and Bolliger CT: Diagnostic yield of bronchoscopy in histologically proven invasive pulmonary aspergillosis. Bone Marrow Transplantation 1999; 24: 1195-9.

6. Hicheri Y, Cook G, and Cordonnier C: Antifungal prophylaxis in hematology patients: the role of voriconazole. Clinical Microbiology and Infection 2012; 18(2): 1-15.

7. Azzola A, Passweg JR, Habicht JM, Bubendorf L, Tamm $\mathrm{M}$, and Gratwohl A: Use of lung resection and voriconazole for successful treatment of invasive pulmonary Aspergillus ustus infection. Journal of Clinical Microbiology 2004; 42(10): 4805-8.

8. Geiser DM, Klich MA, FrisvadJC, Peterson SW, Varga J, and Samson RA: The current status of species recognition and identification in Aspergillus. Studies in Mycology 2007; 59: 1-10.

9. Olorunnipa O, Zhang AY, and Curtin CM: Invasive aspergillosis of the hand caused by Aspergillus ustus: a case report. Hand 2010; 5: 102-5.

10. Cattelan AM, Loy M, Tognon S, Rea F, Sasset L, and Cadrobbi P: Aspergillosis after lung transplantation. Transplant International 2000; 13: 183-6.

11. Zmeili OS and Soubani AO: Pulmonary aspergillosis: a clinical update. Quarterly Journal of Medicine 2007; 100: 317-34.

12. Soubani AO and Chandrasekar PH: The clinical spectrum of pulmonary aspergillosis. Chest 2002; 121(6): 1988-99.

13. Panackal AA, Hong Li, Kontoyiannis DP, Mori M, Perego $\mathrm{CA}$ and Boeckh M: Geoclimatic influences on invasive aspergillosis after hematopoietic stem cell transplantation. Clinical Infectious Diseases 2010; 50(12): 1588-97.

14. Nasim A, Baqi S, Zeeshan SM, and Aziz T: Chronic necrotizing pulmonary aspergillosis in a renal transplant recipient. Journal of Pakistan Medical Association 2011; 61(12): 1242-44.

15. Kousha M, Tadi R and Soubani AO: Pulmonary aspergillosis: a clinical review. European Respiratory Review 2011; 20(121): 156-74.

16. Agarwal R: Allergic bronchopulmonary aspergillosis. Chest 2009; 135(3): 805-26.

17. Cornelis K van der Ent, Hoekstra H, and Rijkers GT: Successful treatment of allergic bronchopulmonary aspergillosis with the recombinant anti- $\operatorname{IgE}$ antibody. Thorax 2007; 62: 276-7.

18. Agarwal K, Chowdhary A, and Gaur SN: A rare case of allergic bronchopulmonary aspergillosis in a patient with chronic obstructive pulmonary disease. Indian Journal of Allergy, Asthma and Immunology 2012; 26(1): 20-4.

19. Denning DW, Riniotis K, Dobrashian R, and Sambatakou $\mathrm{H}$ : Chronic cavitary and fibrosing pulmonary and pleural aspergillosis: case series, proposed nomenclature change, and review. Clin Infectious Diseases 2003; 37(3): 265-80. 
20. Montone KT, LiVolsi VA, Lanza DC, Kennedy DW, Palmer J, and Chiu AG: In-situ hybridization for specific fungal organisms in acute invasive fungal rhinosinusitis. American Journal of Clinical Pathology 2011; 135: 190-9. http://ajcp.oxfordjournals.org/content/ajcpath/135/2/190.fu 11.pdf

21. Stevens DA, Kan VL, Judson MA, Morrison VA, Dummer $\mathrm{S}$ and Denning DW: Practice guidelines for diseases caused by Aspergillus. Clinical Infectious Diseases 2000; 30: 696-709.

22. Yeghen T, Kibbler CC, Prentice HG, Berger LA, Wallesby RK, and McWhinney PHM: Management of invasive pulmonary aspergillosis in hematology patients: A review of 87 consecutive cases at a single institution. Clinical Infectious Diseases 2000; 31: 859-68.

23. Fukuda T, Boeckh M, Carter RA, Sandmaier BM, Maris $\mathrm{MB}$, Maloney DG: Risks and outcomes of invasive fungal infections in recipients of allogeneic hematopoietic stem cell transplants after nonmyeloablative conditioning. Blood 2003; 102(3): 827-33.

24. Kontoyiannis DP, Marr KA, Park BJ, Alexander BD, Anaissie EJ, Walsh TJ: Prospective surveillance for invasive fungal infections in hematopoietic stem cell transplant recipients, 2001-2006: Overview of the Transplant-Associated Infection Surveillance Network (TRANSNET) Database. Clinical Infectious Diseases 2010; 50: 1091-100.

25. Choi JH, Yoo JH, Chung IJ, Kim DW, Han CW, and Shin WS: Esophageal aspergillosis after bone marrow transplant. Bone Marrow Transplantation 1997; 19: 293-4. http://www.nature.com/bmt/journal/v19/n3/pdf/1700629a. pdf

26. Langlois RP, Flegel KM, Meakins JL, Morehouse DD, Robson HG, and Guttmann RD: Cutaneous aspergillosis with fatal dissemination in a renal transplant recipient. Canadian Medical Association Journal 1980; 120: 673-5.

27. Marr KA, Carter RA, Crippa F, Wald A, and Corey L: Epidemiology and outcome of mould infections in hematopoietic stem cell transplant recipients. Clinical Infectious Diseases 2002; 34: 909-17.

28. Denning DW: Commentary: unusual manifestations of aspergillosis. Thorax 1995; 50: 812-3.

29. Law RH and Reyes SA: Primary aspergillosis of the larynx.Case Reports in Otolaryngology 2016; 2016: 1-3.

30. Nahar S, Pillai AK, Kumar JA, Goyal N, and Singh SK: Orbital invasive aspergillosis - A rare fungal infection of the eye. International Journal of Scientific Study 2013; 1(3): $150-4$

31. Walsh TJ, Goodman JL, Pappas P, Bekersky I, Buell DN and Roden M: Safety, tolerance, and pharmacokinetics of high-dose liposomal amphotericin B (AmBisome) in patients infected with Aspergillus species and other filamentous fungi: maximum tolerated dose study. Antimicrobial Agents and Chemotherapy 2001; 45(12): 3487-96.

32. Ascioglu S, Rex JH, de Pauw B, Bennett JE, Bille J, and Crokaert F: Defining opportunistic invasive fungal infections in immunocompromised patients with cancer and hematopoietic stem cell transplants: An international consensus.Clinical Infectious Diseases 2002; 34: 7-14.

33. Pauwa BDe, Walsha TJ, Donnellya JP, Stevens DA, Edwards JE and Calandra T: Fungal infections cooperative group and the National Institute of Allergy and Infectious Diseases Mycoses Study Group (EORTC/MSG) Consensus Group. Clinical Infectious Diseases 2008; 46(12): 1813-21.
34. Franquet T, Muller NL, Gimenez A, Guembe P, Fesus de la Torre and Bague $\mathrm{S}$ : Spectrum of pulmonary aspergillosis: histologic, clinical, and radiologic findings. Radio Graphics 2001; 21: 25-837.

35. Schaffner A, Douglas $H$ and Braude A: Selective protection against conidia by mononuclear and against mycelia by polymorphonuclear phagocytes in resistance to aspergillus. J of Clinical Investigation 1982; 69: 617-31.

36. Garcia-Vidal C, Upton A, Kirby KA and Marr KA: Epidemiology of invasive mold infections in allogeneic stem cell transplant recipients: biological risk factors for infection according to time after transplantation. Clinical Infectious Diseases 2008; 47: 1041-50.

37. Baddley JW, Andes DR, Marr KA, Kontoyiannis DP, Alexander BD, Kauffman CA: Factors associated with mortality in transplant patients with invasive aspergillosis. Clinical Infectious Diseases 2010; 50(12): 1559-67.

38. Stanton LG, George HT, Shelley H, Brian LS, Edward JL and Peter AC: Prolonged granulocytopenia: The major risk factor for invasive pulmonary aspergillosis in patients with acute leukemia. Ann of Inter Med 1984; 100(3): 345-51. http://annals.org/article. aspx ?articleid=698019

39. Ascah KJ, Hyland RH, Hutcheon MA, Urbanski SJ, Pruzanski W and St. Louis EL: Invasive aspergillosis in a healthy patient. Canadian Medical Association Journal 1984; 131: 332-5. http://www.ncbi.nlm.nih.gov/pmc/ articles/PMC1483433/pdf/canmedaj00366-0070.pdf

40. Kulkarni HS: Aspergillosis. American Journal of Respiratory and Critical Care Medicine 2012; 186: 1-2.

41. Kami M, Tanaka Y, Kanda Y, Ogawa S, Masumoto T and Ohtomo K: Computed tomographic scan of the chest, latex agglutination test and plasma $(1 \rightarrow 3)-\beta$-D-glucan assay in early diagnosis of invasive pulmonary aspergillosis: A prospective study of 215 patients. Haematologica 2000; 85(7): 745-52

42. Maertens J, Verhaegen J, Lagrou K, Eldere JV and Boogaerts M: Screening for circulating galactomannan as a noninvasive diagnostic tool for invasive aspergillosis in prolonged neutropenic patients and stem cell trans=plantation recipients: A prospective validation. Blood 2001; 97(6): 1604-10.

43. Sarikaputi M, Morimatsu M, Yamamoto S, Syuto B, Saito $M$ and Naiki M: Latex agglutination test: A simple,rapid and practical method for bovine serum CRP determination. Japanese Journal of Veterinary Research 1992; 40: 1-12.

44. Gupta S: Luminextechnology- innovative flow and bead based technology measuring multiple analytes simultaneously in a single reaction well. U.S.A: Cambridge Biomedical. Available from: http://www. cambridgebiomedical.com/DesktopModules/Bring2mind/ DMX/Download.aspx?Command=Core_Download\&Entry $\mathrm{Id}=2090 \&$ PortalId $=0 \&$ TabId $=155$

45. Landlinger C, Preuner S, Willinger B, Haberpursch B, Racil Z and Mayer J: Species-specific identification of a wide range of clinically relevant fungal pathogens by use of Luminex xMAP technology. Journal of Clinical Microbiology 2009; 47(4): 1063-73.

46. Ya-Ling G, Yi-Qiang C, Wang K, Shou-Ming Q, Cong $\mathrm{W}$ and Jin-Liang K: Accuracy of BAL galactomannanin diagnosing invasive aspergillosis: A bivariate metaanalysis and systematic review. Chest 2010; 138(4): 81724.

47. Arvanitis M, Anagnostou T, Fuchs BB, Caliendo AM and Mylonakisa E: Molecular and nonmolecular diagnostic methods for invasive fungal infections. Clinical Microbiology Reviews 2014; 27(3): 490-526. 
48. Luong ML, Clancy CJ, Vadnerkar A, Kwak EJ, Silveira FP, and Wissel MC: Comparison of an aspergillus realtime polymerase chain reaction assay with galactomannan testing of bronchoalveolar lavage fluid for the diagnosis of invasive pulmonary aspergillosis in lung transplant recipients.Clin Infectious Diseases 2011; 52(10): 1218-26.

49. Barberan J, Candel FJ, and Arribi A: How should we approach Aspergillus in lung secretions of patients with COPD?.Revista Espanola De Quimioterapia 2016; 29(4): 175-82.

50. Becker MJ, Lugtenburg EJ, Cornelissen JJ, Schee CVD, Hoogsteden HC, and Marie SD: Galactomannan detection in computerized tomography- basedbroncho- alveolar lavage fluid and serum in hematological patients at risk for invasive pulmonary aspergillosis. British Journal of Haematology 2003; 121: 448-57.

51. Viscoli C, Machetti M, Cappellano P, Bucci B, Bruzzi P, and Lint MTV: False-positive galactomannan platelia Aspergillus test results for patients receiving piperacillintazobactam. Clinical Infectious Diseases 2004; 38: 913-6.

52. Pfeiffer CD, Fine JP, and Safdar N: Diagnosis of invasive aspergillosis using a galactomannan assay: A metaanalysis. Clinical Infectious Diseases 2006; 42: 1417-27.

53. Maertens J, Raad I, Petrikkos G, Boogaerts M, Selleslag D, and Petersen FB: Efficacy and safety of caspofungin for treatment of invasive aspergillosis in patients refractory to or intolerant of conventional antifungal therapy. Clinical Infectious Diseases 2004; 39: 1563-71.

54. Mennink-Kersten MASH, Ruegebrink D, and Verweij PE: Pseudomonas aeruginosaas a cause of 1,3- $\beta$-D-Glucan assay reactivity. Clin Infectious Dis 2008; 46: 1930-1.

55. Shannon VR, Andersson BS, Lei X, Champlin RE, and Kontoyiannis DP: Utility of early versus late fiberoptic bronchoscopy in the evaluation of new pulmonary infiltrates following hematopoietic stem cell transplantation. Bone Marrow Transplantation 2010; 45: 647-55.

56. Guarner $\mathrm{J}$ and Brandt ME: Histopathologic diagnosis of fungal infections in the $21^{\text {st }}$ century. Clinical Microbiology Reviews 2011; 24(2): 247-80.

57. Liu A: Laser capture microdissection in the tissue biorepository. Journal of Biomolecular Techniques 2010; 21: $120-125$.

58. Kuhlman JE, Fishman EK, Burch PA, Karp JE, Zerhouni EA, and Siegelman SS: CT of invasive pulmonary aspergillosis. American Journal of Roentgenology 1988; 150: 1015-20. Available from: http://www.ajronline.org/ doi/pdf/10.2214/ajr.150.5.1015

59. Jantunen E, Piilonen A, Volin L, Parkkali T, Kahkola PK, and Ruutu T: Diagnostic aspects of invasive Aspergillus infections in allogeneic BMT recipients.Bone Marrow Transplantation 2000; 25: 867-71. Available from: http:// www.nature.com/bmt/journal/v25/n8/pdf/1702232a.pdf

60. Wirk B and Wingard JR:Assessing responses to treatment of opportunistic mycoses salvage strategies.Current Infectious Disease Reports 2011; Available from: https:// www.researchgate.net/profile/Baldeep_Wirk/publication/5 1675247_Assessing_responses_to_treatment_of_opportuni stic_mycoses_and_salvage_strategies/links/00b7d52d41fc 697e5d000000.pdf

61. Kumar R, Chugh $\mathrm{T}$ and Gaur SN: Allergic bronchopulmonary aspergillosis - A review. Indian Journal of Allergy, Asthma and Immunology 2003; 17(2): 55-66.

62. Ward S, Heyneman L, Lee MJ, Leung AN, Hansell DM, and Muller NL: Accuracy of CT in the diagnosis of allergic bronchopulmonary aspergillosis in asthmatic patients. American Journal of Roentgenology 1999; 173:
937-42. Available from: http://www.ajronline.org/doi/pdf/ 10.2214/ajr.173.4.10511153

63. Neofytos D, Horn D, Anaissie E, Steinbach W, Olyaei A, and Fishman J: Epidemiology and outcome of invasive fungal infection in adult hematopoietic stem cell transplant recipients: analysis of multicenter prospective antifungal therapy (PATH) alliance registry. Clinical Infectious Diseases 2009; 48: 265-73.

64. Rijnders BJ, Cornelissen JJ, Slobbe L, Becker MJ, Doorduijn JK, and Hop WCJ: Aerosolized liposomal amphotericin $\mathrm{b}$ for the prevention of invasive pulmonary aspergillosis during prolonged neutropenia: a randomized,placebo-controlled trial. Clinical Infectious Diseases 2008; 46: 1401-8.

65. Miceli $\mathrm{MH}$ and Kauffman CA: Isavuconazole: A new broad-spectrum triazole antifungal agent. Clinical Infectious Diseases 2015; 61(10): 1558-65.

66. Zonios DI, Banacloche JG, Childs R, and Bennett JE: Hallucinations during voriconazole therapy. Clinical Infectious Diseases 2008; 47(1):e7-e10. Available from: https://www.ncbi.nlm.nih.gov/pmc/articles/PMC2727751/

67. Pechlivanoglou P, Le HH, Daenen S, Snowden JA, and Postma MJ: Mixed treatment comparison of prophylaxis against invasive fungal infections in neutropenic patients receiving therapy for hematological malignancies: A systematic review. Journal of Antimicrobial Chemotherapy 2014; 69: 1-11.

68. Muda Z, Ibrahim H, Abdulrahman EJ, Menon BS, Zahari $\mathrm{Z}$ and Zaleha AM: Invasive aspergillosis in pediatric oncology patients. Medical Journal of Malaysia 2008; 63(5): 415-6. Available from: http://www.emjm.org/2008/v63n5/Invasive_Aspergillosis.pdf

69. Nagappan V and Deresinski S: Posaconazole: A broadspectrum triazole antifungal agent. Clinical Infectious Diseases 2007; 45: 1610-7.

70. Walsh TJ, Anaissie EJ, Denning DW, Herbrecht R, Kontoyiannis DP, and Marr KA: Treatment of aspergillosis: Clinical practice guidelines of the infectious diseases society of America. Clinical Infectious Diseases 2008; 46: 327-60.

71. Marr KA, Boeckh M, Carter RA, Kim HW, and Corey L: Combination antifungal therapy for invasive aspergillosis. Clinical Infectious Diseases 2004; 39: 797-802.

72. Cornely OA, Maertens J, Bresnik M, Ebrahimi R, Ullmann AJ and Bouza E: Liposomal amphotericin B as initial therapy for invasive mold infection: A randomized trial comparing a high-loading dose regimen with standard dosing (AmBiLoad Trial). Clinical Infectious Diseases 2007; 44: 1289-97.

73. Denning DW, Park S, Florl CL, Fraczek MG, Kirwan M and Gore R: High-frequency triazole resistance found innonculturable aspergillus fumigatus from lungs of patients with the chronic fungal disease. Clinical Infectious Diseases 2011; 52(9): 1123-9.

74. Cordonnier C, Maury S, Pautas C, Bastie JN, Chehata S, and Castaigne S: Secondary antifungal prophylaxis with voriconazole to adhere to scheduled treatment in leukemic patients and stem cell transplant recipients. Bone Marrow Transplantation 2004; 33: 943-8.

75. Kontoyiannis DP, Hachem R, Lewis RE, Rivero GA, Torres HA, Thornby J: Efficacy and toxicity of caspofungin in combination with liposomal amphotericin $\mathrm{B}$ as primary or salvage treatment of invasive aspergillosis in patients with hematologic malignancies. Cancer 2003; 98(2): 292-9.

76. Olson JA, George A, Constable D, Smith P, Proffitt RT, and Moore JPA: Liposomal amphotericin B and 
echinocandins as monotherapy or sequential or concomitant therapy in murine disseminated and pulmonary Aspergillus fumigatus infections. Antimicrobial Agents and Chemotherapy 2010; 54(9): 3884-94.

77. Olson JA, Schwartz J, Hahka D, George A, Proffitt RT, and Moore JPA: Differences in efficacy and cytokine profiles following echinocandin or liposomal amphotericin B monotherapy or combination therapy for murine pulmonary or systemic Aspergillus flavus infections. Antimicrobial Agents and Chemotherapy 2012; 56(1): 218-30.

78. Walsh TJ, Teppler H, Donowitz GR, Maertens JA, Baden LR, and Dmoszynska A: Caspofungin versus liposomal amphotericin B for empirical antifungal therapy in patients with persistent fever and neutropenia. New England Journal of Medicine 2004; 351: 1391-402.

79. Ullmann AJ, Lipton JH, Vesole DH, Chandrasekar P, Langston A, and Tarantolo SR: Posaconazole or fluconazole for prophylaxis in severe graft-versus-host disease. New England Journal of Medicine 2007; 356(4): 335-47.

80. Baslar Z, Soysal T, Hanc M, Aygun G, Ferhanoglu B, and Sarioglu AC: Successfully treated invasive central nervous system aspergillosis in an allogeneic stem cell transplant recipient. Istanbul (Turkey): the University of Istanbul, Department of Internal Medicine; 2p. Available from: http://www.nature.com/bmt/journal/v22/n4/pdf/1701357a. pdf

81. Prakash G, Thulkar S, Arava SK and Bakhshi S: Cerebral aspergillus infection in pediatric acute lymphoblastic leukemia induction therapy.Indian Journal of Medical and Paediatric Oncology 2012; 33(4): 236-8.

82. Tragiannidis A, Roilides E, Walsh TJ, and Groll AH: Invasive aspergillosis in children with acquired immunodeficiencies. Clin Infectious Diseases 2012; 54(2): 258-67.

83. Tripathi KD: Essentials of Medical Pharmacology.Jaypee Brothers Medical Publishers, New Delhi, Edition $7^{\text {th }}$, 2014: 787-97.

84. Caillot D, Mannone L, Cuisenier B, and Couaillier JF: Role of early diagnosis and aggressive surgery in the management of invasive pulmonary aspergillosis in neutropenic patients. Clinical Microbiology and Infection 2001; 7(2): 54-61.
85. Soltanzadeh H, Wychulis AR, Sadr F, Bolanowski PJ and Neville WE: Surgical treatment of pulmonary aspergilloma. Annals of Surgery 1977; 13-6.

86. Bedi RS: Azoles in allergic bronchopulmonary aspergillosis. Indian Journal of Chest Diseases and Allied Sciences 2015; 57: 5-6.

87. Banh HL: Unconventional treatment options in severe asthma: An overview.Journal of Pharmacy and Pharmaceutical Sciences 2011; 14(3): 387-99.

88. Stevens DA, Schwartz HJ, Lee JY, Moskovitz BL, Jerome DC and Catanzaro A: A randomized trial of itraconazole in allergic bronchopulmonary aspergillosis. New England Journal of Medicine 2000; 342: 756-62.

89. Vora S, Chauhan S, Brummer E and Stevens DA: Activity of voriconazole combined with neutrophils or monocytes against Aspergillus fumigatus: effects of granulocyte colony-stimulating factor and granulocyte-macrophage colony- stimulating factor. Antimicrobial Agents and Chemotherapy1998; 42(9): 2299-303.

90. Floral CL, Izquierdo AA, Estrella MC, Perkhofer S and Tudela JLR: In-vitro activities of various antifungal drugs against Aspergillus terreus: Global Assessment Using the Methodology of the European Committee on Antimicrobial Susceptibility Testing. Antimicrobial Agents and Chemotherapy 2009; 53(2): 794-5. http://aac. asm.org/content/53/2/794.full.pdf+html

91. Bader O, Weig M, Reichard U, Lugert R, Kuhns M and Christner M: cyp51A-based mechanisms of Aspergillus fumigatus azole drug resistance present in clinical samples from Germany. Antimicrobial Agents and Chemotherapy 2013; 57(8): 3513-17.

92. Chamilos G, Marom EM, Lewis RE, Lionakis MS and Kontoyiannis DP: Predictors of pulmonary zygomycosis versus invasive pulmonary aspergillosis in patients with cancer. Clinical Infectious Diseases 2005; 41: 60-6.

93. Rickerts V, Mousset S, Lambrecht E, Tintelnot K, Schwerdtfeger $\mathrm{R}$ and Presterl E: Comparison of histopathological analysis, culture, and polymerase chain reaction assays to detect invasive mold infections from biopsy specimens. Clinical Infectious Diseases 2007; 44: 1078-83.

94. Nucci M and Perfect JR: When primary antifungal therapy fails. Clinical Infectious Diseases 2008; 46: 1426-33.

How to cite this article:

Paul D and Paul K: Aspergillosis: An overview. Int J Pharm Sci \& Res 2018; 9(12): 5032-49. doi: 10.13040/IJPSR.0975-8232.9(12).5032-49.

All @ 2013 are reserved by International Journal of Pharmaceutical Sciences and Research. This Journal licensed under a Creative Commons Attribution-NonCommercial-ShareAlike 3.0 Unported License.

This article can be downloaded to ANDROID OS based mobile. Scan QR Code using Code/Bar Scanner from your mobile. (Scanners are available on Google Play store) 